\title{
Myeloid cell heterogeneity in lung cancer: implication for immunotherapy
}

\author{
Sabina Sangaletti ${ }^{1} \cdot$ Roberto Ferrara $^{2} \cdot$ Claudio Tripodo $^{3,4} \cdot$ Marina Chiara Garassino $^{2} \cdot$ Mario Paolo Colombo $^{1}$ (i)
}

Received: 9 November 2020 / Accepted: 13 March 2021 / Published online: 2 April 2021

(c) The Author(s), under exclusive licence to Springer-Verlag GmbH Germany, part of Springer Nature 2021

\begin{abstract}
Lung is a specialized tissue where metastases from primary lung tumors takeoff and those originating from extra-pulmonary sites land. One commonality characterizing these processes is the supportive role exerted by myeloid cells, particularly neutrophils, whose recruitment is facilitated in this tissue microenvironment. Indeed, neutrophils have important part in the pathophysiology of this organ and the key mechanisms regulating neutrophil expansion and recruitment during infection can be co-opted by tumor cells to promote growth and metastasis. Although neutrophils dominate the myeloid landscape of lung cancer other populations including macrophages, dendritic cells, mast cells, basophils and eosinophils contribute to the complexity of lung cancer TME. In this review, we discuss the origin and significance of myeloid cells heterogeneity in lung cancer, which translates not only in a different frequency of immune populations but it encompasses state of activation, morphology, localization and mutual interactions. The relevance of such heterogeneity is considered in the context of tumor growth and response to immunotherapy.
\end{abstract}

Keywords Lung cancer $\cdot$ Myeloid cells $\cdot$ DNA-based traps $\cdot$ Immunotherapy

\section{Myeloid cell heterogeneity in lung cancer}

Definition of myeloid cell heterogeneity encompasses diversity of composition, function and state of activation of myeloid cells. However, morphology and location are two less considered source of heterogeneity, which might reflect peculiar myeloid cell activities.

Sabina Sangaletti and Roberto Ferrara have equally contributing to this work.

Mario Paolo Colombo

mariopaolo.colombo@istitutotumori.mi.it

1 Department of Research, Molecular Immunology Unit, Fondazione IRCCS Istituto Nazionale Dei Tumori, via Amadeo 42, 20133 Milano, Italy

2 Department of Medical Oncology, Fondazione IRCCS Istituto Nazionale dei Tumori, Milano, Italy

3 Tumor Immunology Unit, University of Palermo, Palermo, Italy

4 FIRC Institute of Molecular Oncology (IFOM), Milano, Italy

\section{State of activation and composition}

Current immunology describes a great variety of immune cells each one characterized by additional variability in terms of phenotype and state of activation. This heterogeneity not only characterizes T-cells and B-cells but also myeloid cells.

Among myeloid cells macrophages are peculiar examples of immune heterogeneity. Their heterogeneity or functional plasticity is associated to the capacity of intercepting and adapting to the different tissue-specific signals or pathologic conditions [1]. Recently Ballesteros and coll. have shown that such "tissue-specific adaptation" also occurs in neutrophils that, although characterized for short survival, can acquire heterogeneity at the chromatin, RNA, and receptor levels when recruited in dedicated micro anatomical niches [2].

These transcriptome diversities are intercepted by single-cell RNA-sequencing (scRNA-seq). Zilionis and coll. mapped myeloid cells in non-small-cell lung cancer patients describing more than 20 conserved myeloid cell types, in part reproducible across patients [3]. Differences might depend on the extreme variability of the samples considered: adenocarcinomas (LA) vs. squamous cell carcinoma 
(SCC), male vs. female, and tumor stages. In their analysis, they found 3 dendritic cell (DC) and 1 plamacytoidDC populations, 3 monocyte and 9 macrophage sub-types, 5 different neutrophil subsets and 2 mast cell types. Notably, macrophages were clearly distinct from monocytes, but poorly defined within the M1 and M2 clusters, rather most of them exhibited a non-distinct M2-like phenotype and were referred as belonging to a peculiar subset of alveolar macrophages. The different cytokines composition and gradient characterizing the tumor context, might generate a continuum of different states of activation [3]. A concept that can be extended to dendritic cells and neutrophils, which in the lung cancer TME, are present in four and five distinct subsets respectively. For DC, two subsets were clearly identified as conventional DC type 1 and type 2 that are either efficient antigen cross-presenters to $\mathrm{CD} 8+\mathrm{T}$ cells or committed for interaction with CD4 $+\mathrm{T}$ cells, respectively. The third subset displayed a LPS-like activated phenotype and the last subset was of plasmacytoid DC. Among neutrophils, 4 populations expressed canonical neutrophil markers and were distinguished based on their maturation steps. A fifth population expressing type- I interferon genes was considered a distinct subset. These sc-RNA seq data were also informative in showing that myeloid cells of peripheral blood do not entirely overlap with those identified in the tumor, supporting their plasticity in adapting to different tissue requirements including those undergoing neoplastic transformation [3].

Sc-RNA approaches becomes even more important when used to evaluate transcriptional changes in the cancer-associated microenvironment induced by therapeutics. In a recent work Maynard et al. collected samples from metastatic lung cancer patients before and during targeted therapy for singlecell RNA sequencing-based analyses. Comparing the gene expression profiles of cells from progressive disease (PD) with baseline samples they found more than 900 upregulated genes [4]. Changes were both in tumor cells and their associate immune microenvironment. Baseline and PD TME samples were enriched in macrophages that however differed in state of activation being either associated with M2 phenotype or IDO expression, respectively. Differently, responder patients had a prominent hot TME with expression of proinflammatory genes and activated $\mathrm{T}$ cells [4].

\section{Morphology}

An unexpected determinant of myeloid heterogeneity also reflecting peculiar functions is the myeloid cell morphology. Within the NSCLCs TME, macrophages can display a prominent elongated or stellate morphology, which is prominent in cells infiltrating the tumor stroma, or monocytoid or display epithelioid shape mostly in macrophages intermingling with tumor cells (Fig. 1). Among epithelioid histiocytes, multinucleated forms can be detected (Fig. 1 arrow). These elements have been classically associated with persistent immune stimulation by specific pathogens (e.g. mycobacteria) or inflammatory noxae $[5,6]$. Epithelioid macrophages have a prominent endoplasmic reticulum, which is suggestive of high secretory activity, which found positive correlation with the increased collagen synthesis and extracellular matrix remodeling of involved tissues. Very recently, we described that a quite similar macrophage subset characterizes the TME of nearly $20 \%$ of NSCLC patients who, treated with immune checkpoint inhibitors (ICI), developed hyperprogressive disease (HPD), a paradoxical boost in tumor growth [7]. In tumors from these patients, epithelioid macrophages formed clusters that could be identified through the co-espression of CD163, CD33 and PD-L1 markers. Interestingly, when HPD was modeled in mice, epithelioid macrophages were identified for the expression of CD206 and aggregated in fibrotic- collagen rich areas of HP tumors. Mechanistically, FcR triggering of clustered epithelioid macrophages by ICI Abs delivered a signaling cascade promoting pro-tumorigenic functional reprogramming. This indicates that the presence of epithelioid macrophages could represent a tumor microenvironment feature associated with ICI failure [7].

\section{Location}

Another factor that might contribute to myeloid cell heterogeneity in NSCLC is their topography. Reciprocal spatial distribution of immune cells within a TME is potential biomarkers for successful immunotherapy in patients with solid tumors [8]. Indeed, using in vivo imaging, Arlauckas SP and coll. showed that the spatial association between macrophages and CD8 T-cell was responsible for resistance to a-PD1 being the a-PD-1 mAbs, initially binding PD-1 on tumor-infiltrating $\mathrm{CD} 8+\mathrm{T}$ cells captured, within minutes, by tumor- associated macrophages via their Fc $\gamma$ Rs [9].

Location of myeloid cells in the TME can be influenced by different factors, including chemokines produced by tumor and stromal cells. In the lung TME, malignant cancer cells can reprogram the tumor-infiltrating stromal cells, which in turn contribute to carcinogenesis either directly or though further conditioning the nearby immune cells.

The diversity of stromal cells in NSCLC TME is little considered but rather relevant for myeloid cell distribution. Figure 2 shows that different stromal cells composition can be associated with variable degree of myeloid and T-cell infiltration and/or with their PD-L1 expression. Smooth muscle and fibroblast-rich stroma is associated to high tumor PD-L1, high stromal T-cells and low myeloid cells. On the contrary stroma rich in myofibroblasts and fibroblasts was associated with high myeloid cell infiltration and low T-cells. 


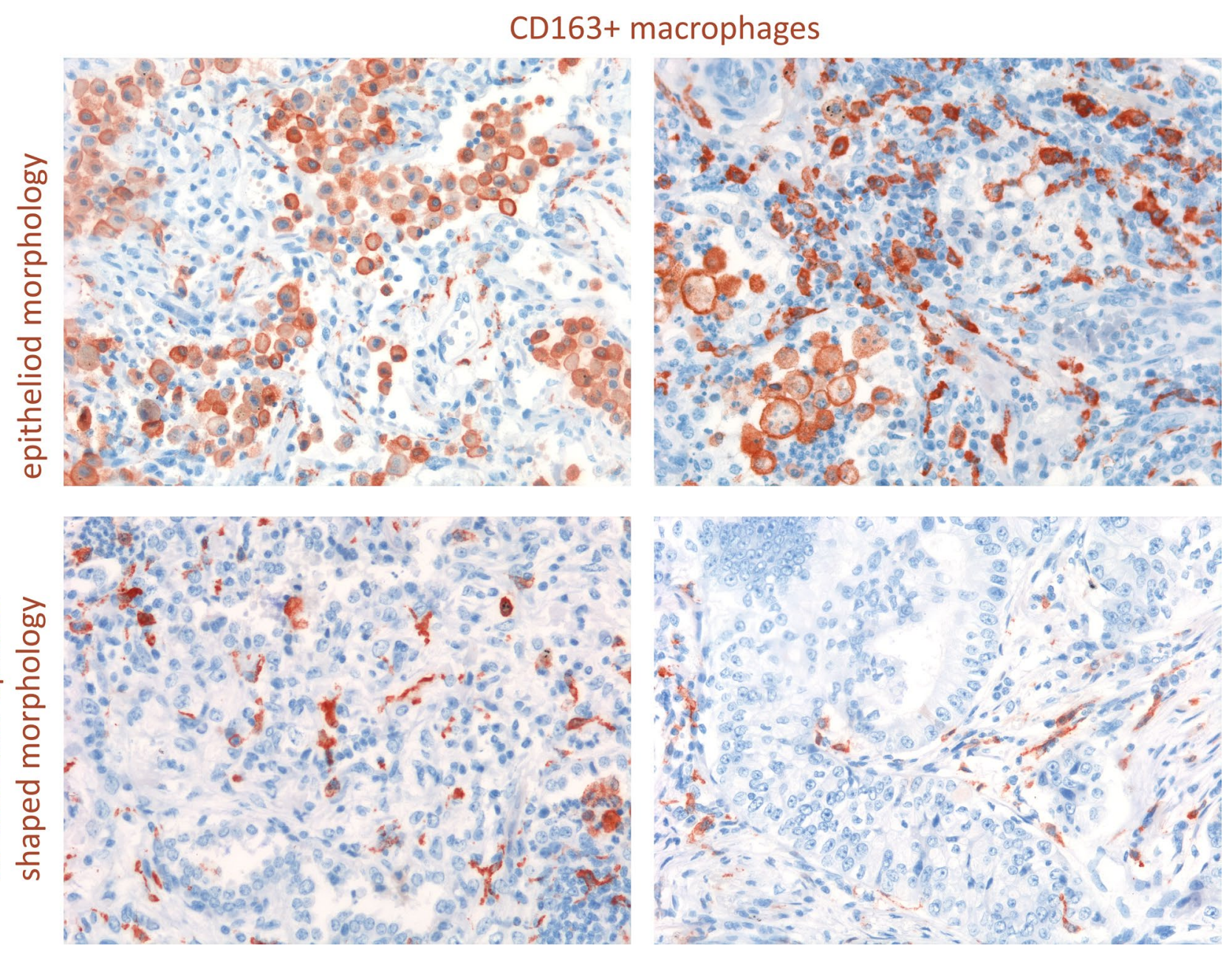

Fig. 1 In NSCLC microenvironment macrophages can display a prominent elongated or stellate morphology, mostly in cells infiltrating the tumor stroma, or monocytoid and epitheliod shape often observed in macrophages intermingling with tumor cells. Arrow show multinucleated cells detected among epithelioid histiocytes
Although in a completely different tumor context such as diffuse large B-cell lymphoma, we have recently shown that different mesenchymal populations can differently modulate the expression of key transcriptional pathway involved in lymphoma outgrowth such as Myc or damage response programs and immune checkpoints.

All these data should be interpreted in light of the underestimated immune modulatory role of mesenchymal cells [10] that only in part has been characterized in solid tumors for the possible impact on immune therapy.

\section{Neutrophils dominate the lung cancer myeloid landscape}

Although a variety of myeloid populations characterize lung cancer TME, neutrophils dominate this peculiar landscape $[11,12]$. Reasons for such predominance can be found in the physiology of this organ, which is "primed" toward neutrophil recruitment and expansion. The respiratory system is continually exposed to microorganisms and one of the most important components of the early innate response is the vigorous recruitment of neutrophils. Bacteria initially interact with alveolar epithelial cells and macrophages, which respond with the secretion of cytokines and neutrophil chemo-attractants. The lung microvasculature is a site for marginated neutrophils to immediately respond to and capture bloodstream pathogens. Interestingly, differently from the spleen or liver, which are dominated by macrophage-mediated host defense the lung microvascular host defense is dominated by neutrophils [13]). Since 1920, it has been observed that bacterial infection of lower respiratory tract induces the recruitment of morphologically immature neutrophils from the bone marrow into circulation, an event recognized as "left shift". A similar and evident left shift characterized also viral infection, for example a comprehensive flow cytometry of 


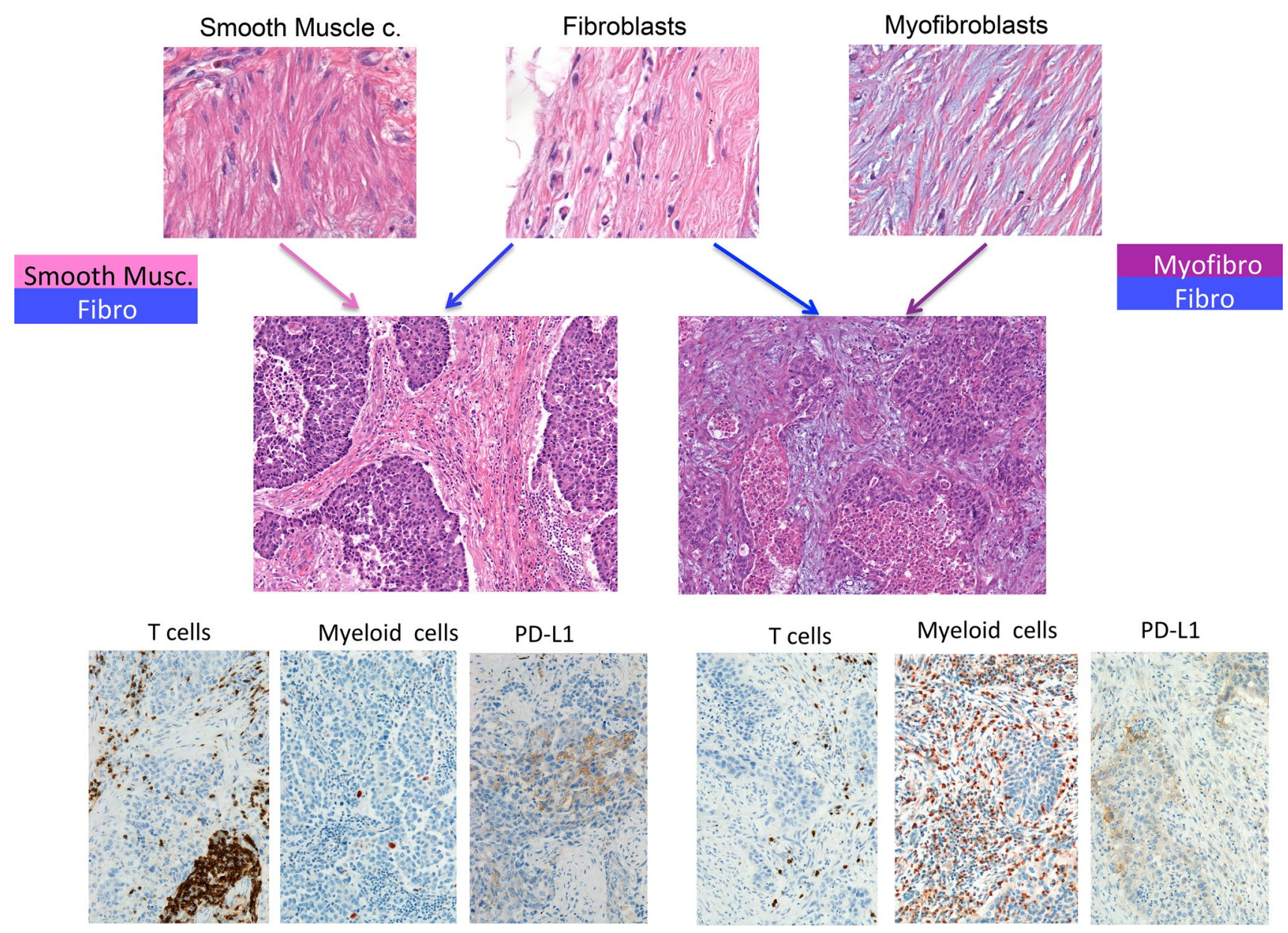

Fig. 2 Stroma composition determines T-and myeloid-cell infiltration in lung adenocarcinoma. Smooth muscle and fibroblast-rich stroma is associated to high tumor PD-L1, stromal T-cell infiltra-

whole blood samples from 54 COVID-19 patients revealed increased immature neutrophils in correlation with disease severity [14]. Similarly the expansion of these immature populations has been shown in NSCLC cancer patients, an event now called leukemoid reaction [15].

This evidence suggests that lung cancer can co-opt mechanisms proper infection to exacerbate a vicious tumor promoting loop. Leukemoid reaction is common to several solid tumors. Granger and coll. evaluated 3770 consecutive cases and found that $10 \%$ of them were characterized by extreme leukocytosis. Among those cases, 53\% of patients overall had tumors involving the lungs (including metastasis from other sites) and $17 \%$ of them had non-small cell lung cancer (NSCLC). This suggests that the lung microenvironment could predispose to the development of leukemoid reaction, independently from the tumor origin. In lung cancer $66 \%$ of patients diagnosed with leukemoid reaction died within 12 weeks [16] and similar dismal prognosis has tion and low myeloid cells. On the contrary a stroma rich in myofibroblasts and fibroblasts is associated with high myeloid cell infiltration and low T-cells

been reported by Kasuga and coll. [17] that also correlated leukemoid reaction with high G-CSF in serum.

The expansion of immature neutrophils can be easily detected in the peripheral blood. Indeed, immature "low density " neutrophils remain in the PBMC layer during Ficoll gradient separation [18] and, if analyzed by FACS, they clearly show the lack of CD10 and CD16 expression [19]. The relevance of this population is mainly due to its immune suppressive properties on T-cell proliferation, as shown in preclinical model [20]. Notably low-density granulocyte population comprises human myeloid derived suppressor cells that are more commonly characterized, as HLADR-, CD33 + and CD11b +, and are a mixture of monocytic and granulocytic subpopulations. Polymorphonuclear (PMN) MDSCs are HLA-DR ${ }^{-} \mathrm{CD} 14^{-} \mathrm{CD} 15^{+}$ or $\mathrm{CD} 6 \mathrm{~b}^{+}$, whereas monocytic (M) MDSC are HLA-DR ${ }^{-/ \text {low }} \mathrm{CD} 14^{+} \mathrm{CD}^{-} 5^{-}[21]$.

The negative impact of immature neutrophils in the TME is also metabolic and due to their capacity to 
overcome nutrient limitations and to suppress anti-tumor immunity in the glucose deprived TME. These "oxidative neutrophils" are able to maintain NADPH-oxidase dependent ROS production in the absence of glucose usage through fatty acid dependent mitochondrial function [22].

\section{Extrusion of DNA-based traps}

Immune suppression in not the only way through which neutrophils may have an impact on lung cancer spread. Indeed, the extrusions of DNA-based traps (NET) have been recently suggested to play a relevant role. Whether NET extrusion is a feature of mature or immature neutrophils is still debated, indeed although NET are formed by mature neutrophils as part of their anti-microbial functions some groups have reported NET extruded by MDSCs or immature cells [23, 24].

In 2004 a pathologist was the first is showing that neutrophils can extrude spider web-like chromatin structures to entrap bacteria, which were called neutrophil extracellular traps [25]. Extracellular chromatin is composed by dsDNA and histones also decorated with anti-microbial proteins like myeloperoxidase (MPO) and neutrophil elastase [25]. After their discovering, several types of innate immune cells have also been reported to extrude their DNA, the list includes eosinophils [26] macrophages [27], mast cells [28] but also cells of the adaptive arm of the immune system like B-cells [29] and CD4 T-helper cells [30]. NET play a direct pathogenic role in promoting tissue damages [31] and auto (ANCA)-antibodies development in systemic vasculitis [32] and lupus erythematous [33]. Many different papers are now describing NET in the context of cancer [34-36]. Using murine models of TNBC, Park et al. showed that NET stimulate invasion and migration of breast cancer cells. Inhibiting NET formation or digesting NET with DNAse I in vivo reduced lung metastasis [37]. Using pre-clinical murine models of lung and colon cancer in combination with intravital video microscopy, Rayes and coll. showed that NET functionally regulate disease progression and that blocking NETosis through multiple strategies significantly inhibits spontaneous metastasis to the lung and liver [38]. Similarly, Najmeh using the A549 lung cancer cell line showed that NET facilitate liver metastasis through 1 integrin mediated adhesion to tumor cells [39]. Furthermore, NET induced in the lung by tobacco smoke exposure or nasal instillation of LPS awaken dormant cancer cells and convert them into growing metastases [40]. In an interesting paper Cools-Lartigue and coll. correlated infection conditions with microvascular NET deposition and the consequent pro-metastatic trapping of circulating lung carcinoma cells [41]. Whether a local infection, which is a frequent condition in lung cancer patients, could influence neutrophil trap formation in the lung and tumor growth is unclear. When tested in vitro upon
PMA stimulation, neutrophils from healthy and LC patients had the same capacity to extrude NET [42]. Therefore, it is likely that tumor-specific conditions (i.e. cytokine production) or local infections or hypoxia [43] can impact on NET extrusion and therefore on cancer progression.

\section{Mechanisms underlying myeloid cell heterogeneity in lung cancer}

Within NSCLCs, lung adenocarcinoma is characterized by high mutational diversity [44] also associated with high stroma cell variability. Indeed, driver mutations might promote tumor-intrinsic pathway activating specific immune infiltration and local immune suppression [45]. The activation of Myc in KRAS-G12D mutant mice, promotes tumor aggressiveness through the recruitment of macrophages and the reduction of $\mathrm{T}$ and B-cells infiltration via CCL9 and IL23, respectively [46]. KRAS or HRAS mutants increase the stability of PDL1mRNA via MEK activation and induce PDL1 expression through phosphorylated ERK (pERK) signaling [47]. Similarly, in EGFR mutant the activation of pERK pathways upregulated PDL1 expression and therefore immune suppression [48]. The additional loss of the tumor suppressor LKB1 in KRAS-mutant mouse tumors is associated with increased accumulation of immunosuppressive neutrophils, exhausted T cells, increased pro- inflammatory cytokines, including interleukin-6 (IL-6), decreased PDL1 expression and reduction of IFN + T-cells [49].

Finally, in addition to the known driving mutations that might impact on immune infiltration, multi-omics approaches applied to multiple loci, showed another level of complexity due to the existence of heterogeneous mutations leading to immunological hot and cold areas, within a single tumor [50]. Interestingly such increased antigenicity did not correlate with T-cell cytotoxicity that was rather decreased through a feedback loop resulting in the local recruitment of immune suppressive cells.

\section{Myeloid cells in response to immune checkpoint inhibitors (ICI) in lung cancer}

ICI have changed fundamentally the treatment paradigm of NSCLC patients. Anti-PD-1/PD-L1 agents have shown improved responses and survival benefit when given as single agent or in combination with either chemotherapy or anti-CTLA4, in first line setting [51]. However, progression rate with anti-PD-1/PD-L1 in combination with chemotherapy or anti-CTLA4 is $15 \%[52,53]$ and may rise to $40 \%$ [54] in patients treated with a single agent either PD-1 or PD-L1 inhibitor. In addition, HPD upon ICI has been reported between $13.8 \%$ [55] and up to $37 \%$ [56] of 
NSCLC suggesting that specific adaptive [57] or innate [7] immune cells may predispose a subgroup of patients to increased tumor growth upon PD-1/PD-L1 inhibitors. Myeloid cells are able to sustain cancer stem cells [58] to promote immune evasion as well as to induce resistance to systemic treatment. Circulating neutrophils and monocytes have been associated with lack of benefit from ICI in advanced NSCLC patients [59]. Absolute neutrophils count (ANC), neutrophil to lymphocyte ratio (NLR) and derived neutrophil lymphocytes ratio (dNLR) correlate with cancer associated inflammation, and are considered active players of disease progression and poor survival in several solid tumors. Baseline ANC $\geq 7500 / \mu \mathrm{L}$ correlates with worse overall survival (OS) [hazard ratio $(\mathrm{HR})=3.46$, $\mathrm{p}=0.03]$ and progression free survival (PFS) $(\mathrm{HR}=3.97$, $\mathrm{p}=0.001)$ in advanced NSCLC patients treated with nivolumab [60]. Similarly, both pretreatment NLR $\geq 5$ [61] and the dynamic monitoring of the ratio between pre and post anti-PD1 treatment NLR [62] significantly correlate with worse survival outcomes $(\mathrm{HR}=1.43, \mathrm{p}=0.04)$ upon nivolumab. dNLR [absolute neutrophil count/(white blood cell count-absolute neutrophil count)] may be more relevant than NLR because it includes monocytes and other granulocyte subpopulations. dNLR has been associated to worse OS $(\mathrm{HR}=1.70, \mathrm{p}<0.001)$ in advanced NSCLC treated with ICI but not with chemotherapy [63]. Furthermore, dNLR $>4$ significantly correlates with HPD upon ICI in a retrospective series of NSCLC patients [64]. Considering these results, NLR and/or dNLR have been included in several indexes and prognostic models and represent useful tools to stratify NSCLC patients' risk of progression before ICI initiation [65] [66]. Despite most of the current evidences on circulating neutrophils has been reported regarding ICI treated patients, dNLR has been described as a poor prognostic factor also in NSCLC patients treated with cytotoxic chemotherapy $[67,68]$ or tyrosine kinase inhibitors [68], suggesting that cancer related inflammation reflected by circulating myeloid parameters is associated with worse survival regardless of treatment type.

Absolute monocytes count (AMC) and lymphocyte to monocyte ratio (LMR) have also been associated with worse outcome upon anti-PD-1/PD-L1 agents in NSCLC patients. In fact, post-nivolumab AMC was higher in nonresponders compared to responders [69], similarly, high baseline LMR was a good predictor of response in NSCLC patients upon nivolumab.

Besides blood parameters, a more detailed analysis of myeloid cell subpopulations by flow cytometry is of paramount significance to characterize the mechanisms beyond ICI resistance. In this regard, immature neutrophils identified by FACS through the lack of CD10 and CD16 [70] or CD15 + CD16- [71] immature neutrophils were both associated with rapid progression upon ICI in advanced NSCLC patients.

Circulating human MDSCs have also been correlated with poor prognosis upon ICI. Human MDSCs express markers of myeloid lineage, as CD33 and CD11b, and are a mixture of monocytic and granulocytic subpopulations. Polymorphonuclear (PMN) MDSCs are HLA$\mathrm{DR}^{-} \mathrm{CD} 14^{-} \mathrm{CD} 15^{+}$or $\mathrm{CD}_{66 b^{+}}$, whereas monocytic (M) MDSC are HLA-DR ${ }^{-/ \text {low }} \mathrm{CD} 14^{+} \mathrm{CD} 15^{-}$[21]. Early accumulation of M-MDSC expressing the immunomodulatory galectin-9 was related to primary and secondary resistance to nivolumab in metastatic NSCLC patients through the impairment of IFN-y secretion by CD8 + T cells [72]. Similarly, PMN-MDSCs expressing lectin-type oxidized LDL receptor (Lox-1) or high levels of chemokine and soluble factors capable of MDSC recruitment and proliferation were significantly higher in NSCLC with no response to nivolumab therapy [73].

Future studies in search of myeloid circulating biomarkers predicting response to ICI will consider the immunometabolic features of innate immune cells. In this regard, circulating neutrophils with an immature phenotype are capable of oxidative metabolism and of inducing T-cell immune suppression through radical oxygen species (ROS) production [22].

Although most biomarkers of response to ICI treatment on tissue samples were related to adaptive immune compartment, a recent retrospective analysis has shown an epigenetic signature, called EPIMMUNE, which correlates with PFS in advanced NSCLC patients treated with antiPD1 agents. In particular, EPIMMUNE negative tumors were enriched in macrophages and neutrophils and were prevalent among non-responders, on the contrary, EPIMMUNE positive biopsies were infiltrated by lymphocytes and were more frequent among responders [74]. However, some subtypes of myeloid cells commonly promote antitumor immunity, as observed for a M1 macrophages whose signature has been associated with durable clinical benefit upon anti-PD1 in NSCLC patients [75]. Similarly, PD-1/ PD-L1 axis on macrophages can induce a non-inflammatory non-phagocytic state that could be reversed by antiPD-1/PD-L1 agents [76]. These findings suggest that non only T-lymphocytes but also myeloid cells are directly affected by ICI.

As for circulating biomarkers, a comprehensive characterization tumor infiltrating myeloid cells by multiparametric flow cytometry, multidimensional IHC or single cell RNA sequencing are urgently needed to provide new and in deep knowledge on the role of innate immunity in shaping response to ICI in NSCLC patients. 


\section{Targeting myeloid cells in lung cancer}

Given the impact of myeloid cells on the response to immunotherapy in lung cancer, testing chemokine and cytokine pathways involved into the recruitment and maintenance of these immunosuppressive cells might offer new hints to design new drug combinations with ICI.

Tumor associated macrophages (TAM) and MDSC remain the preferential target for this approach [77]. In fact, although potentially promising, neutrophil targeting strategies may have limited clinical application. As an example, depleting neutrophils may increase the risk of severe infections, on the other hand, reprogramming neutrophil functions from pro-tumor to antitumor may be associated with increased inflammation and tissue damage [78]. Different preclinical mouse models have tested the possibility of targeting MDSCs. In the 3LL model, gemcitabine, cimetidine and anti-Ly6G Ab depleted MDSC recruitment hampering tumor growth, while increasing NK and CD8 T cell activity. Resveratrol or CCL2 antagonists were effective in reducing both recruitment and immune suppressive function of MDSC in the $3 \mathrm{LL}$ model (reviewed in [79]). On the same line Merad and coll. used single-cell RNAseq and mass spectrometry to identify chemokine and cytokine pathways involved in the recruitment and maintenance of MDSCs, validated the relevance of these pathways in preclinical studies and are now designing a neoadjuvant "window-of-opportunity" trial to evaluate the synergy of PD-1 blockade with disruption of CCR $2 / 5$ or interleukin- 8 mediated myeloid recruitment in early-stage NSCLC lesions [80].

Chemotherapy has the potential to eliminate myeloid cells and their detrimental effect during ICI treatment. Indeed, preclinical data obtained using 5-FU and gemcitabine in preclinical mouse models have shown a positive effect of these chemotherapeutic agents in inducing MDSC apoptosis. However, other compounds, such as doxorubicin or high-dose cyclophosphamide, have been shown to increased MDSC number and suppressive activity. Ding et al. showed that cyclophosphamide promotes the expansion of suppressive monocytic MDSC [81]. This event was the result of a feedback regulatory loop aiming at restoring myeloid cell level, after the initial depletion induced by chemotherapy, and to inhibit the inflammatory condition elicited by chemotherapy. Therefore after an initial "reset" of the tumor microenvironment, chemotherapy-induced inflammatory responses may re-enforce MDSC recruitment and suppressive functions and worsen the therapeutic outcome.

Besides chemotherapy also antiangiogenic drugs influence myeloid cell functions. In a clinical trial on stage IV NSCLC patients with EGFR mutation, Bevacizumab was effective in controlling intracranial lesions when used in combination with TKI inhibitors. The analysis of myeloid and $\mathrm{T}$ cells showed that such combination was effective in reducing the level of circulating S100A9 + MDSCs while increasing CD8 and Th1 signatures [82]. A similar study in NSCLC patients by Koinis F. et al. showed that bevacizumab-based chemotherapy significantly reduced the levels of granulocytic MDSCs [83]. However, an increase in the levels of CD15-positive monocytic MDSCs was associated with poor response to treatment and disease progression.

Although TAMs are a promising target in the treatment of lung cancer, no drugs have been used so far in patients.

Differently selective macrophages targeting agents are currently being tested in several cancer types. MARCO and CSF1/CSF1R axis are other promising targets able to reprogram or inhibit TAM recruitment within the TME in different solid tumor models [84] and preliminary results of the anti-CSFR 1 antibody cabiralizumab and nivolumab have been reported in PDAC patients [85]. However, CSF1R inhibition is an example of compensatory immune crosstalk as a mechanism of resistance to immune targeting agents. In fact, CSF1R is highly expressed also by cancer associated fibroblasts and its blockade on these cells induce a significant MDSC and neutrophils recruitment within the tumor site limiting antitumor responses. Triple blockade with CSFR-1 inhibitors, anti -PD1 agents and CXCR2 antagonist can bypass this crosstalk and inhibit tumor growth in preclinical models [86]. Similarly, dual targeting of CXCR2 + neutrophils and CCR2 + TAM increased antitumor immunity and response to cytotoxic chemotherapy in PDAC models [87] suggesting that double or triple blockade may counteract compensatory immunological bypass tracks.

A possible warning on strategies aiming to block TAM recruitment comes from data showing a possible detrimental rebound after CCL2 blockade. Bonapace et al. showed in mice that CCL2 blockade was effective in reducing TAM and limiting tumor growth however cessation of the therapy stimulates their quick rebound within the tumors leading to accelerated metastatic disease also promoting angiogenesis [88]. Overall these data suggest that eliminating or decreasing macrophages/myeloid cells could not be a rational approach if leading to a rebound effect. By contrast, 'reeducating' macrophages could be preferred as a strategic approach to improve immunotherapy [89].

Funding This work was supported by AIRC (IG no. 22204 to SS and IG no. 18425 to MPC), Ministero della Salute ( Young Research Grant no. GR-2013-02355637 to SS), IASLC Young Investigator Award 2019. 


\section{Compliance with ethical standards}

Conflict of interest SS, CT and MPC have no conflict of Interest to declare. Dr. Roberto Ferrara reports personal fees from MSD (advisory board). Dr. Marina Chiara Garassino reports grants and personal fees from Eli Lilly, Otsuka Pharma, Astra Zeneca, Novartis, BMS, Roche, from Pfizer, Celgene, Incyte, Inivata, Bayer, MSD, GlaxoSmithKline S.p.A, Sanofi-Aventis, Spectrum Pharmaceutcials, Blueprint Medicine; personal fees from Seattle Genetics, Daiichi Sankyo, Boehringer Ingelheim, Takeda, Janssen, Mirati Therapeutics; grants from United Therapeutics Corporation, Turning Point, Merck Serono, Tiziana Sciences, Ipsen, MedImmune, Exelisis, Array (Pfizer), Clovis; non-financial support from MSD, EliLilly.

Ethica approval Tumor specimens were collected from patients with NSCLC treated with ICI at the Thoracic Unit of the Istituto Nazionale dei Tumori, Milan.The study complied with the Declaration of Helsinki and was done in accordance with good clinical practice guidelines. All samples were obtained according to the Internal Review and the Ethics Boards of the Istituto Nazionale Tumori of Milan and all patients provided informed consent. All experimental protocols were approved by the ethics boards of the Istituto Nazionale Tumori of Milan (Int 22/15). Author Contribution SS, RF \&MPC wrote the review, CT selected images and assembled the Figure, MG reviewed the manuscript.

\section{References}

1. Natoli G, Ostuni R (2019) Adaptation and memory in immune responses. Nat Immunol. 20:783-792. https://doi.org/10.1038/ S41590-019-0399-9

2. BallesterosIR-P A, Genua M, Lusito E, Kwok I, Fernández-Calvo G, Khoyratty TE, Grinsven E, González-Hernández S, NicolásÁvila JA, Vicanolo T, Maccataio A, Benguría A, Li JL, Adrover JM, Aroca-Crevillen A, Quintana JA, Martín-Salamanca S, Mayo F, Ascher S, Barbiera G, Soehnlein O, Gunzer M, Ginhoux F, Sánchez-Cabo F, Nistal-Villán E, Schulz C, Dopazo A, Reinhardt C, Udalova IA, Ng LG, Ostuni R, Hidalgo A (2020) Co-option of neutrophil fates by tissue environments. Cell 183:1282-1297. https://doi.org/10.1016/j.cell.2020.10.003

3. Zilionis R, Engblom C, Pfirschke C et al (2019) Single-cell transcriptomics of human and mouse lung cancers reveals conserved myeloid populations across individuals and species. Immunity 50:1317. https://doi.org/10.1016/j.immuni.2019.03.009

4. Maynard A, McCoach CE, Rotow JK et al (2020) TherapyInduced Evolution of Human Lung Cancer Revealed by SingleCell RNA Sequencing. Cell 182:1232. https://doi.org/10.1016/j. cell.2020.07.017

5. Ramakrishnan L (2012) Revisiting the role of the granuloma in tuberculosis. Nat Rev Immunol 12:352-366. https://doi.org/10. 1038/nri3211

6. Turk JL, Narayanan RB (1982) The origin, morphology, and function of epithelioid cells. Immunobiology 161:274-282.

7. Lo Russo G, Moro M, Sommariva M et al (2019) Antibody-Fc/ $\mathrm{FcR}$ interaction on macrophages as a mechanism for hyperprogressive disease in non-small cell lung cancer subsequent to PD-1/ PD-L1 blockade. Clin Cancer Res 25:989-999. https://doi.org/10. 1158/1078-0432.Ccr-18-1390

8. Kather JN, Suarez-Carmona M, Charoentong P et al (2018) Topography of cancer-associated immune cells in human solid tumors. Elife 7:e36967. https://doi.org/10.7554/eLife.36967

9. Arlauckas SP, Garris CS, Kohler RH et al (2017) In vivo imaging reveals a tumor-associated macrophage-mediated resistance pathway in anti-PD-1 therapy. Sci Transl Med. https://doi.org/10. 1126/scitranslmed.aal3604

10. Ricciardi M, Zanotto M, Malpeli G, Bassi G, Perbellini O, Chilosi M, Bifari F, Krampera M (2015) Epithelial-to-mesenchymal transition (Emt) elicits mesenchymal stromal cell-like immune modulatory properties in cancer cells. Cytotherapy 17:S20.

11. Stankovic B, Bjorhovde HAK, Skarshaug R et al (2019) Immune cell composition in human non-small cell lung cancer. Front Immunol 9:3101. https://doi.org/10.3389/fimmu.2018.03101

12. Kargl J, Busch SE, Yang GHY et al (2017) Neutrophils dominate the immune cell composition in non-small cell lung cancer. Nat Commun 8:14381. https://doi.org/10.1038/ncomms14381

13. Granton E, Kim JH, Podstawka J, Yipp BG (2018) The lung microvasculature is a functional immune Niche. Trends Immunol 39:890-899. https://doi.org/10.1016/j.it.2018.09.002

14. Carissimo G, Xu WL, Kwok I et al (2020) Whole blood immunophenotyping uncovers immature neutrophil-to-VD2 T-cell ratio as an early marker for severe COVID-19. Nat Commun 11:5243.

15. Craig A, Mai J, Cai SS, Jeyaseelan S (2009) Neutrophil recruitment to the lungs during bacterial pneumonia. Inf Immun 77:568575. https://doi.org/10.1128/Iai.00832-08

16. Granger JM, Kontoyiannis DP (2009) Etiology and outcome of extreme leukocytosis in 758 nonhematologic cancer patients a retrospective single-institution study. Cancer Am Cancer Soc 115:3919-3923. https://doi.org/10.1002/cncr.24480

17. Kasuga I, Makino S, Kiyokawa H, Katoh H, Ebihara Y, Ohyashiki K (2001) Tumor-related leukocytosis is linked with poor prognosis in patients with lung carcinoma. Cancer Am Cancer Soc. 92:2399-2405.

18. Hassani M, Hellebrekers $\mathrm{P}$, Chen N, van Aalst C, Bongers S, Hietbrink F, Koenderman L, Vrisekoop N (2020) On the origin of low-density neutrophils. J Leukocyte Biol 107:809-818.

19. Marini O, Costa S, Bevilacqua D et al (2017) Mature CD10(+) and immature CD10(-) neutrophils present in G-CSF-treated donors display opposite effects on T cells. Blood 129:1343-1356. https://doi.org/10.1182/blood-2016-04-713206

20. Sagiv JY, Michaeli J, Assi S et al (2015) Phenotypic diversity and plasticity in circulating neutrophil subpopulations in cancer. Cell Rep 10:562-573. https://doi.org/10.1016/j.celrep.2014.12.039

21. Bronte V, Brandau S, Chen SH et al (2016) Recommendations for myeloid-derived suppressor cell nomenclature and characterization standards. Nat Commun 7:12150. https://doi.org/10.1038/ ncomms 12150

22. Rice CM, Davies LC, Subleski JJ et al (2018) Tumour-elicited neutrophils engage mitochondrial metabolism to circumvent nutrient limitations and maintain immune inhibition. J Immunol 9:1-13

23. Alfaro C, Teijeira A, Onate C et al (2016) tumor-produced interleukin-8 attracts human myeloid-derived suppressor cells and elicits extrusion of neutrophil extracellular traps (NETs). Clin Cancer Res 22:3924-3936. https://doi.org/10.1158/1078-0432. Ccr-15-2463

24. Sangaletti S, Talarico G, Chiodoni C et al (2019) SPARC is a new myeloid-derived suppressor cell marker licensing suppressive activities. Front Immunol 10:1369. https://doi.org/10.3389/fimmu. 2019.01369

25. Brinkmann V, Reichard U, Goosmann C, Fauler B, Uhlemann Y, Weiss DS, Weinrauch Y, Zychlinsky A (2004) Neutrophil extracellular traps kill bacteria. Science 303:1532-1535

26. Yousefi S, Simon D, Kozlowski E, Schmid I, Simon HU (2008) Eosinophils release mitochondrial DNA to trap and kill bacteria. Swiss Med Wkly. 138:43

27. Chow OA, von Kockritz-Blickwede M, Bright AT et al (2010) Statins enhance formation of phagocyte extracellular traps. Cell Host Microbe. 8:445-454. https://doi.org/10.1016/j.chom.2010. 10.005 
28. Lin AM, Rubin CJ, Khandpur R et al (2011) Mast cells and neutrophils release IL-17 through extracellular trap formation in psoriasis. J Immunol 187:490-500. https://doi.org/10.4049/jimmunol. 1100123

29. Ingelsson B, Soderberg D, Strid T et al (2018) Lymphocytes eject interferogenic mitochondrial DNA webs in response to $\mathrm{CpG}$ and non-CpG oligodeoxynucleotides of class C. Proc Nat Acad Sci United States Am 115:E 478-E487. https://doi.org/10.1073/pnas. 1711950115

30. Costanza M, Poliani PL, Portararo P et al (2019) DNA threads released by activated CD4(+) T lymphocytes provide autocrine costimulation. Proc Nat Acad Sci United States Am 116:89858994. https://doi.org/10.1073/pnas. 1822013116

31. Kessenbrock K, Krumbholz M, Schonermarck U, Back W, Gross WL, Werb Z, Grone HJ, Brinkmann V, Jenne DE (2009) Netting neutrophils in autoimmune small-vessel vasculitis. Nat Med 15:623-625

32. Sangaletti S, Tripodo C, Chiodoni C et al (2012) Neutrophil extracellular traps mediate transfer of cytoplasmic neutrophil antigens to myeloid dendritic cells toward ANCA induction and associated autoimmunity. Blood 120:3007-3018. https://doi.org/10.1182/ blood-2012-03-416156

33. Lande R, Ganguly D, Facchinetti V et al (2011) Neutrophils activate plasmacytoid dendritic cells by releasing self-DNA-peptide complexes in systemic lupus erythematosus. Sci Trans. Med 3:73

34. Cedervall J, Olsson AK (2016) Immunity gone astray-NETs in cancer forum. Trends Cancer 2:633-634. https://doi.org/10. 1016/j.trecan.2016.10.012

35. Sangaletti S, Tripodo C, Vitali C et al (2014) Defective stromal remodeling and neutrophil extracellular traps in lymphoid tissues favor the transition from autoimmunity to lymphoma. Cancer Discov 4:110-129. https://doi.org/10.1158/2159-8290.Cd-13-0276

36. Brinkmann V (2018) Neutrophil extracellular traps in the second decade. J Innate Immun 10:414-421. https://doi.org/10.1159/ 000489829

37. Park J, Wysocki RW, Amoozgar Z et al (2016) Cancer cells induce metastasis-supporting neutrophil extracellular DNA traps. Sci Trans Med 8:361. https://doi.org/10.1126/scitranslmed.aag1711

38. Rayes RF, Mouhanna JG, Nicolau I et al (2019) Primary tumors induce neutrophil extracellular traps with targetable metastasispromoting effects. Jci Insight 4. https://doi.org/10.1172/jci.insig ht. 128008

39. Najmeh S, Cools-Lartigue J, Rayes RF et al (2017) Neutrophil extracellular traps sequester circulating tumor cells via 1-integrin mediated interactions. Int J Cancer 140:2321-2330. https://doi. org/10.1002/ijc.30635

40. Albrengues J, Shields MA, Ng D et al (2018) Neutrophil extracellular traps produced during inflammation awaken dormant cancer cells in mice. Science 361:1353. https://doi.org/10.1126/science. aao4227

41. Cools-Lartigue J, Spicer J, McDonald B, Gowing S, Chow S, Giannias B, Bourdeau F, Kubes P, Ferri L (2013) Neutrophil extracellular traps sequester circulating tumor cells and promote metastasis. J Clin Invest 123:3446-3458. https://doi.org/10.1172/ Jci67484

42. Arpinati L, Shaul ME, Kaisar-Iluz N, Mali S, Mahroum S, Fridlender ZG (2020) NETosis in cancer: a critical analysis of the impact of cancer on neutrophil extracellular trap (NET) release in lung cancer patients vs. mice. Cancer Immunol Immun 69:199213. https://doi.org/10.1007/s00262-019-02474-x

43. Branitzki-Heinemann K, Mollerherm H, Vollger L et al (2016) Formation of neutrophil extracellular traps under Low oxygen Level. Front Immunol 7:518. https://doi.org/10.3389/fimmu.2016. 00518

44. Altorki NK, Markowitz GJ, Gao DC, Port JL, Saxena A, Stiles B, McGraw T, Mittal V (2019) The lung microenvironment: an important regulator of tumour growth and metastasis. Nat Rev Cancer 19:9-31. https://doi.org/10.1038/s41568-018-0081-9

45. Wellenstein MD, de Visser KE (2018) cancer-cell-intrinsic mechanisms shaping the tumor immune landscape. Immunity 48:399416. https://doi.org/10.1016/j.immuni.2018.03.004

46. Kortlever RM, Sodir NM, Wilson CH, Burkhart DL, Pellegrinet L, Swigart LB, Littlewood TD, Evan GI (2017) Myc cooperates with Ras by programming inflammation and immune suppression. Cell 171:1301. https://doi.org/10.1016/j.cell.2017.11.013

47. Coelho MA, Trecesson SD, Rana S et al (2017) Oncogenic RAS signaling promotes tumor immunoresistance by stabilizing PD-L1 mRNA. Immunity 47:1083. https://doi.org/10.1016/j.immuni. 2017.11.016

48. Chen N, Fang WF, Zhan JH et al (2015) Upregulation of PD-L1 by EGFR activation mediates the immune escape in EGFR-driven NSCLC implication for optional immune targeted therapy for NSCLC patients with EGFR mutation. J Thorac Oncol. 10:910 923. https://doi.org/10.1097/Jto.0000000000000500

49. Koyama S, Akbay EA, Li YY et al (2016) STK11/LKB1 deficiency promotes neutrophil recruitment and proinflammatory cytokine production to suppress T-cell activity in the lung tumor microenvironment. Cancer Res 76:999-1008. https://doi.org/10. 1158/0008-5472.Can-15-1439

50. Jia QZ, Wu W, Wang YQ et al (2018) Local mutational diversity drives intratumoral immune heterogeneity in non-small cell lung cancer. Nat Commun 9:5361. https://doi.org/10.1038/ s41467-018-07767-w

51. Proto C, Ferrara R, Signorelli D et al (2019) Choosing wisely first line immunotherapy in non-small cell lung cancer (NSCLC): what to add and what to leave out. Cancer Treat Rev 75:39-51. https:// doi.org/10.1016/j.ctrv.2019.03.004

52. Gandhi L, Rodriguez-Abreu D, Gadgeel S et al (2018) Pembrolizumab plus chemotherapy in metastatic non-small-cell lung cancer. New Engl J Med 378:2078-2092. https://doi.org/10.1056/ NEJMoa1801005

53. Hellmann MD, Ciuleanu TE, Pluzanski A et al (2018) Nivolumab plus ipilimumab in lung cancer with a high tumor mutational burden. New Engl J Med 378:2093-2104. https://doi.org/10.1056/ NEJMoa1801946

54. Rittmeyer A, Barlesi F, Waterkamp D et al (2017) Atezolizumab versus docetaxel in patients with previously treated non-small-cell lung cancer (OAK): a phase 3, open-label, multicentre randomised controlled trial. Lancet 389:255-265. https://doi.org/10.1016/ S0140-6736(16)32517-X

55. Ferrara R, Mezquita L, Texier M et al. Hyperprogressive Disease in Patients With Advanced Non-Small Cell Lung Cancer Treated With PD-1/PD-L1 Inhibitors or With Single-Agent Chemotherapy. JAMA Oncology 4(11):1543

56. Kim CG, Kim KH, Pyo KH et al (2019) Hyperprogressive disease during PD-1/PD-L1 blockade in patients with non-small-cell lung cancer. Ann Oncol 30:1104-1113. https://doi.org/10.1093/ annonc/mdz123

57. Ferrara R, Naigeon M, Auclin E et al (2021) Circulating T-cell Immunosenescence in Patients with Advanced Non-small Cell Lung Cancer Treated with Single-agent PD-1/PD-L1 Inhibitors or Platinum-based Chemotherapy. Clin Cancer Res 2021; 27:492503. https://doi.org/10.1158/1078-0432.CCR-20-1420

58. Komura N, Mabuchi S, Shimura K et al (2020) The role of myeloid-derived suppressor cells in increasing cancer stem-like cells and promoting PD-L1 expression in epithelial ovarian cancer. Cancer Immunol Immun.

59. Prelaj A, Tay R, Ferrara R, Chaput N, Besse B, Califano R (2019) Predictive biomarkers of response for immune checkpoint inhibitors in non-small-cell lung cancer. Eur J Cancer 106:144-159. https://doi.org/10.1016/j.ejca.2018.11.002 
60. Tanizaki J, Haratani K, Hayashi H et al (2018) Peripheral blood biomarkers associated with clinical outcome in non-small cell lung cancer patients treated with Nivolumab. J Thorac Oncol 13:97-105. https://doi.org/10.1016/j.jtho.2017.10.030

61. Bagley SJ, Kothari S, Aggarwal C et al (2017) Pretreatment neutrophil-to-lymphocyte ratio as a marker of outcomes in nivolumabtreated patients with advanced non-small-cell lung cancer. Lung Cancer 106:1-7. https://doi.org/10.1016/j.lungcan.2017.01.013

62. Suh KJ, Kim SH, Kim YJ, Kim M, Keam B, Kim TM, Kim DW, Heo DS, Lee JS (2018) Post-treatment neutrophil-to-lymphocyte ratio at week 6 is prognostic in patients with advanced non-small cell lung cancers treated with anti-PD-1 antibody. Cancer Immunol Immun 67:459-470. https://doi.org/10.1007/s00262-017-2092-x

63. Mezquita L, Auclin E, Ferrara R et al (2018) Association of the lung immune prognostic index with immune checkpoint inhibitor outcomes in patients with advanced non-small cell lung cancer. Jama Oncol 4:351-357. https://doi.org/10.1001/jamaoncol.2017.4771

64. Kim Y, Kim CH, Lee HY et al (2019) Comprehensive clinical and genetic characterization of hyperprogression based on volumetry in advanced non-small cell lung cancer treated with immune checkpoint inhibitor. J Thorac Oncol 14:1608-1618. https://doi.org/10. 1016/j.jtho.2019.05.033

65. Park W, Mezquita L, Okabe N et al (2020) Association of the prognostic model iSEND with PD-1/L1 monotherapy outcome in nonsmall-cell lung cancer. Brit J Cancer 122:340-347. https://doi.org/ 10.1038/s41416-019-0643-y

66. Prelaj A, Ferrara R, Rebuzzi SE et al (2019) EPSILoN: A Prognostic Score for Immunotherapy in Advanced Non-Small-Cell Lung Cancer: A Validation Cohort. Cancers 11:1954. https://doi.org/10. 3390/cancers 11121954

67. Charrier M, Mezquita L, Lueza B et al (2019) Circulating innate immune markers and outcomes in treatment-naive advanced nonsmall cell lung cancer patients. Eur J Cancer 108:88-96. https://doi. org/10.1016/j.ejca.2018.12.017

68. Kazandjian D, Gong YT, Keegan P, Pazdur R, Blumenthal GM (2019) Prognostic value of the lung immune prognostic index for patients treated for metastatic non-small cell lung cancer. Jama Oncol 5:1481-1485. https://doi.org/10.1001/jamaoncol.2019.1747

69. Khunger M, Patil PD, Khunger A et al (2018) Post-treatment changes in hematological parameters predict response to nivolumab monotherapy in non-small cell lung cancer patients. PLoS ONE 13:e0197743. https://doi.org/10.1371/journal.pone.0197743

70. Ferrara R, Lo Russo G, Signorelli D et al (2020) Circulating and tumor-associated neutrophil subtypes discriminate hyperprogressive disease (HPD) from conventional progression (PD) upon immune checkpoint inhibitors (ICI) in advanced non-small cell lung cancer (NSCLC) patients (pts) and in vivo models. J Clin Oncol 38:9547

71. Mezquita L, Martin-Romano P, Auclin E et al (2019) Circulating immature neutrophils, tumor-associated neutrophils and dNLR for identification of fast progressors to immunotherapy in NSCLC. J Thorac Oncol 14:S272-S273.

72. Limagne E, Richard C, Thibaudin M, Fumet JD, Truntzer C, Lagrange A, Favier L, Coudert B, Ghiringhelli F (2019) Tim-3/ galectin-9 pathway and mMDSC control primary and secondary resistances to PD-1 blockade in lung cancer patients. Oncoimmunology 8:e1564505. https://doi.org/10.1080/2162402X.2018.1564505

73. Kim HR, Park SM, Seo SU et al (2019) The ratio of peripheral regulatory T cells to Lox-1(+) polymorphonuclear myeloid-derived suppressor cells predicts the early response to anti-PD-1 Therapy in patients with non-small cell lung cancer. Am J Resp Crit Care 199:243-246. https://doi.org/10.1164/rccm.201808-1502LE

74. Duruisseaux M, Martinez-Cardus A, Calleja-Cervantes ME et al (2018) Epigenetic prediction of response to anti-PD-1 treatment in non-small-cell lung cancer: a multicentre, retrospective analysis. Lancet Resp Med 6:771-781. https://doi.org/10.1016/S22132600(18)30284-4
75. Riaz N, Havel JJ, Makarov V et al (2017) Tumor and microenvironment evolution during immunotherapy with Nivolumab. Cell 171:934. https://doi.org/10.1016/j.cell.2017.09.028

76. Hartley GP, Chow L, Ammons DT, Wheat WH, Dow SW (2018) Programmed cell death ligand 1 (PD-L1) signaling regulates macrophage proliferation and activation. Cancer Immunol Res 6:12601273. https://doi.org/10.1158/2326-6066.Cir-17-0537

77. Colombo MP, Mantovani A (2005) Targeting myelomonocytic cells to revert inflammation-dependent cancer promotion. Cancer Res 65:9113-9116. https://doi.org/10.1158/0008-5472.Can-05-2714

78. Shaul ME, Fridlender ZG (2019) Tumour-associated neutrophils in patients with cancer. Nat Rev Clin Oncol 16:601-620. https://doi. org/10.1038/s41571-019-0222-4

79. Yang ZZ, Guo JC, Weng LL, Tang WX, Jin SL, Ma W (2020) Myeloid-derived suppressor cells-new and exciting players in lung cancer. J Hematol Oncol 13:10. https://doi.org/10.1186/ s13045-020-0843-1

80. Marron TU, Leader A, Lavin Y et al (2020) Targeting Myeloid Cells that Define the Tumor Immune Microenvironment in NSCLC. J Thorac Oncol 15:S5

81. Ding ZC, Munn DH, Zhou G (2014) Chemotherapy-induced myeloid suppressor cells and antitumor immunity: the Janus face of chemotherapy in immunomodulation. Oncoimmunology 3. https:// doi.org/10.4161/21624011.2014.954471

82. Feng PH, Chen KY, Huang YC et al (2018) Bevacizumab reduces S100A9-positive MDSCs linked to intracranial control in patients with EGFR-mutant lung adenocarcinoma. J Thorac Oncol 13:958967. https://doi.org/10.1016/j.jtho.2018.03.032

83. Koinis F, Vetsika EK, Aggouraki D, Skalidaki E, Koutoulaki A, Gkioulmpasani M, Georgoulias V, Kotsakis A (2016) Effect of firstline treatment on myeloid-derived suppressor cells' subpopulations in the peripheral blood of patients with non-small cell lung cancer. J Thorac Oncol 11:1263-1272. https://doi.org/10.1016/j.jtho.2016. 04.026

84. Georgoudaki AM, Prokopec KE, Boura VF et al (2016) Reprogramming tumor-associated macrophages by antibody targeting inhibits cancer progression and metastasis. Cell Rep 15:2000-2011. https:// doi.org/10.1016/j.celrep.2016.04.084

85. Wang-Gillam A, O'Reilly EM, Bendell JC et al (2019) A randomized phase II study of cabiralizumab (cabira) plus nivolumab (vivo) $+/$ - chemotherapy (chemo) in advanced pancreatic ductal adenocarcinoma (PDAC). J Clin Oncol 37:670. https://doi.org/10. 1200/JCO.2019.37.4_suppl.TPS465

86. Kumar V, Donthireddy L, Marvel D et al (2017) Cancer-associated fibroblasts neutralize the anti-tumor effect of CSF1 receptor blockade by inducing PMN-MDSC infiltration of tumors. Cancer Cell 32:654. https://doi.org/10.1016/j.ccell.2017.10.005

87. Nywening TM, Belt BA, Cullinan DR et al (2018) Targeting both tumour-associated CXCR2(+) neutrophils and CCR2(+) macrophages disrupts myeloid recruitment and improves chemotherapeutic responses in pancreatic ductal adenocarcinoma. Gut 67:1112. https://doi.org/10.1136/gutjnl-2017-313738

88. Bonapace L, Coissieux MM, Wyckoff J, Mertz KD, Varga Z, Junt T, Bentires-Alj M (2014) Cessation of CCL2 inhibition accelerates breast cancer metastasis by promoting angiogenesis. Nature 515 . https://doi.org/10.1038/nature13862

89. Guiducci C, Vicari AP, Sangaletti S, Trinchieri G, Colombo MP (2005) Redirecting in vivo elicited tumor infiltrating macrophages and dendritic cells towards tumor rejection. Cancer Res 65:3437-3446.

Publisher's Note Springer Nature remains neutral with regard to jurisdictional claims in published maps and institutional affiliations. 